\title{
Structural changes introduced by cholesterol and melatonin to the model membranes mimicking preclinical conformational diseases
}

\author{
Tatiana Murugova ${ }^{1,2}$, Oleksandr Ivankov ${ }^{1,2,3}$, Elena Ermakova ${ }^{1}$, Tomáš Kondela ${ }^{1,4,5}$, \\ Pavol Hrubovčák ${ }^{1,6}$, Vadim Skoi ${ }^{1,2}$, Alexander Kuklin ${ }^{1,2}$ and Norbert Kučerka ${ }^{1,4}$ \\ ${ }^{1}$ Frank Laboratory of Neutron Physics, Joint Institute for Nuclear Research, Dubna, Russia \\ ${ }^{2}$ Moscow Institute of Physics and Technology, Dolgoprudny, Russia \\ ${ }^{3}$ Institute for Safety Problems of Nuclear Power Plants NAS of Ukraine, Kyiv, Ukraine \\ ${ }^{4}$ Department of Physical Chemistry of Drugs, Faculty of Pharmacy, Comenius University in Bratislava, Bratislava, Slovakia \\ ${ }^{5}$ Department of Nuclear Physics and Biophysics, Faculty of Mathematics, Physics and Informatics, Comenius University in \\ Bratislava, Bratislava, Slovakia \\ ${ }^{6}$ Department of Condensed Matter Physics, University of P. J. Šafárik in Košice, Košice, Slovakia
}

\begin{abstract}
The structure and dynamics of membranes depend on many external and internal factors that in turn determine their biological functions. One of the widely accepted and studied characteristics of biomembranes is their fluidity. We research a simple system with variable fluidity tweakable via its composition. The addition of cholesterol is employed to increase the order of lipid chains, thus decreasing the membrane fluidity, while melatonin is shown to elevate the chain disorder, thus also the membrane fluidity. We utilize the densitometric measurements to show a shift of studied systems closer or further from the gel-to-fluid phase transition. The structural changes represented by changes to membrane thickness are evaluated from small angle neutron scattering. Finally, we look at the ability of the two additives to control the interactions between membrane and amyloid-beta peptides. Our results suggest that fluidizing effect of melatonin can promote an insertion of peptide within the membrane interior. Intriguingly, the latter structure relates possibly to an Alzheimer's disease preventing mechanism postulated in the case of melatonin.
\end{abstract}

Key words: Membrane fluidity - Cholesterol - Melatonin — Amyloid-beta peptide - Neutron scattering

Abbreviations: AD, Alzheimer's disease; DOPC, 1,2-dioleoyl-sn-glycero-3-phosphocholine; DMPC, 1,2-dimyristoyl-sn-glycero-3-phosphocholine; $\mathrm{A} \beta$, amyloid-beta; SANS, small angle neutron scattering.

\section{Introduction}

About $50 \%$ of the brain's dry weight is lipid, supporting the various structural or metabolic functions taking place therein (Lim and Wenk 2009). A huge diversity of lipid types included in a delicate balance regulates structural properties of biomembranes (Kučerka et al. 2015). However, the lipids of different shape and size do not affect the

Correspondence to: Norbert Kučerka, Frank Laboratory of Neutron Physics, Joint Institute for Nuclear Research, 141980 Dubna, Russia E-mail: kucerka@nf.jinr.ru static structure only, but their different chemical composition modulates also the elasto-mechanical properties of membrane. The membrane fluidity is one of the examples that have been a topic of vast number of studies (Petrache et al. 1998; Nagle and Tristram-Nagle 2000; Chu et al. 2005; Martinez-Seara et al. 2008; Kučerka et al. 2009; Ermilova and Lyubartsev 2019). In addition, the membrane properties have been shown to be affected by the incorporation of other small molecules, such as cholesterol and melatonin (Drolle et al. 2013; Choi et al. 2014; Dies et al. 2014). The role of membrane properties, its chemical composition and additives is thus not surprisingly discussed in a connection 
with supporting the membrane biofunctions as well as various disfunctions. Amongst other biological processes studied in this regard recently, is also amyloid toxicity manifesting during conformational diseases (Martel et al. 2017; Ntarakas et al. 2019).

One of the conformational diseases challenging the humankind is the Alzheimer's disease (AD) that is a devastating neurodegenerative disease characterized by dementia and memory loss. It is estimated that 47 million people around the world suffer from dementia and with increasing ageing of population this number will increase. It is estimated this number will reach 131 millions by the year 2050 (Prince et al. 2016). A fingerprint characteristic to $A D$ is the formation of senile plaques, primarily consisting of amyloid- $\beta(\mathrm{A} \beta)$ peptides. The main role in the formation of the plaques, especially their initiation, may be played by the interactions between peptides and membrane (Murphy 2007). While the peptide aggregates form outside the membrane, capability of membrane to retain peptides within its interior could be understood as a preventive mechanism to the disease onset. Not surprisingly, the composition of membranes has been shown to modulate these interactions. For example, cholesterol in elevated concentrations appears to inhibit the insertion of peptide into the membrane (Dante et al. 2006), that is in the agreement with its hypothesised preference for the fluid membranes (Ahyayauch et al. 2012). Nevertheless, the addition of melatonin as a fluidizing agent was surprisingly found to also reduce the population of the membraneembedded A $\beta$ state (Dies et al. 2014). The inconsistencies in the literature results then corroborate the complexity of the system, in which the other factors such as dehydration, curvature effect, and electrostatic interactions most likely came into the play in the mentioned studies. It is thus interesting to revisit the effect of elasto-mechanical properties while excluding the role of other influences.

The primary effect of cholesterol (Fig. 1A) on the structural properties of membrane is the increasing order of lipid hydrocarbon chains and thus the overall stiffness of membrane (Rog et al. 2009). Melatonin (Fig. 1B) on the other hand, has completely different effect. It has been shown to increase the fluidity of membrane and counteract the influence of cholesterol (Drolle et al. 2013; Choi et al. 2014; Reiter et al. 2014). The opposite effects of the two molecules therefore suggest naturally to be utilized in a regulation of membrane physical properties and perhaps in a control of amyloid fibril formation and toxicity. Moreover, both of the molecules are present in the brain, contained in the membranes at concentrations up to $33 \%$ in the case of cholesterol (Zhang and Liu 2015). The melatonin levels have been reported with a high variability, that is likely affected by the high permeability of cell membranes resulting in its equivalent distribution between inter- and extra-cellular compartments (Yu et al. 2016). Nevertheless, the presence of lipophilic moieties in addition to those of hydrophilic nature, makes melatonin to partition preferentially to the lipid bilayer at its interface with water (Ceraulo et al. 1999). The biological relevance of melatonin is supported also by the fact that ageing is one significant factor of decreasing production of melatonin which reduces its potential role in preventing the development of $\mathrm{AD}$ among older population (Karasek 2004).

We are extending the previous studies that looked at the possibility to regulate the membrane fluidity by the addition of cholesterol and melatonin (Drolle et al. 2013; Choi et al. 2014; Dies et al. 2014). We focus on the simplified model system without anionic lipids to eradicate the effect of electrostatic interactions. In addition, the membranes are dispersed in water solution to enforce the full hydration conditions without any artifacts due to dehydration. After corroborating the stiffening and fluidizing effects of cholesterol and melatonin, respectively in the case of simple model membrane, we examine further how these effects are modulated in the presence of amyloid-beta peptides.

\section{Materials and Methods}

\section{Sample preparation}

1,2-dioleoyl-sn-glycero-3-phosphocholine (DOPC) and 1,2-dimyristoyl-sn-glycero-3-phosphocholine (DMPC) were purchased from Avanti Polar Lipids (Alabaster, AL). Melatonin and cholesterol were obtained from SigmaAldrich (St. Louis, MO). Amyloid-beta peptide segment 25-35, $A \beta_{25-35}$, was purchased from Abbiotec (Escondido, CA). All chemicals were in powder form and used without further purification.
A

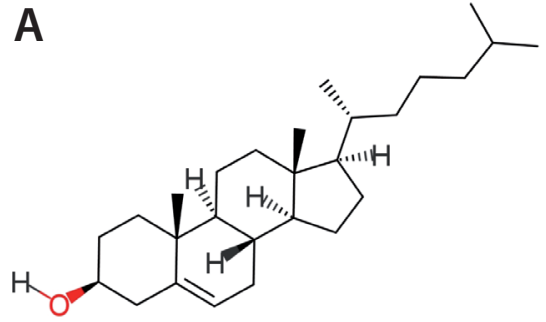<smiles>COc1ccc2[nH]cc(CCNC(C)=O)c2c1</smiles>

Figure 1. Schematics of the chemical structure of cholesterol (A) and melatonin (B). 
Lipids were dissolved in chloroform in glass vials at the total concentration of $25 \mathrm{mg} / \mathrm{ml}$, and melatonin and cholesterol at concentrations $3 \mathrm{mg} / \mathrm{ml}$. The peptide was subjected to pretreatment procedure (Barrett et al. 2015) for its disaggregation, and dissolved in chloroform:trifluoroethanol solvent mixture (1:1) at concentration $6 \mathrm{mg} / \mathrm{ml}$. Components at the desired ratios were mixed in microtubes and evaporated to dryness. The remaining solvent was removed under vacuum for 12 hours. The samples contained varying amounts of cholesterol and/or melatonin up to $29 \mathrm{~mol} \%$ that proved to avoid the bilayer destabilization and cholesterol crystallization (Huang et al. 1999; Kučerka et al. 2008b). The peptide was added to the selected samples at the concentration of $3 \mathrm{~mol} \%$. The low peptide concentration was used to diminish the possibility of its spontaneous aggregation and bilayer structure disruption (Dies et al. 2014).

The samples for densitometry measurements were mixed with $\mathrm{H}_{2} \mathrm{O}$ (Millipore, $18 \mathrm{M} \Omega \mathrm{cm}$ ) and exposed to several freeze-thaw cycles resulting in homogeneous suspensions of multilamellar vesicles (MLVs). The small angle neutron scattering (SANS) measurements on the other hand, were performed with samples of unilamellar vesicle (ULVs) prepared in $\mathrm{D}_{2} \mathrm{O}$ containing water $(100 \%, 75 \%$, and $50 \%)$ in order to increase neutron scattering length contrast between hydrogen-rich lipid and deuterium-rich water (i.e., $\mathrm{D}_{2} \mathrm{O}$ ). In addition, decreasing the amount of hydrogen in sample, which is a strong source of incoherent scattering (i.e., high scattering background), serves the improvement of signalto-noise ratio in neutron scattering experiments. The ULVs were prepared by extrusion through polycarbonate filters with 500 Å pores (Avanti Polar Lipids, Alabaster, AL). The total mass concentration (lipid+cholesterol+melatonin) was identical in all samples and equaled to $10 \mathrm{mg} / \mathrm{ml}$. This concentration provides satisfactory scattering intensities, while guaranteeing sufficient water between ULVs to eliminate the effect of multiple scattering and possibility of interparticle interactions (Kučerka et al. 2007).

\section{Densitometry}

The densities of our membrane systems were measured utilizing vibrating-tube density meter DMA-5000 (Anton-Paar, Graz, Austria). The measuring principle of the instrument is based on the change of the natural frequency of a hollow $\mathrm{U}$ tube oscillator when filled with different liquids or gases. The additional mass of the sample changes its natural frequency due to a gross mass change of the oscillator. The direction of oscillation is perpendicular to the plane of the U-shaped sample tube. The frequency of the oscillator is influenced only by the volume fraction which is in the vibrating part of the sample tube. Since the vibrating volume is always the same inside the sample tube, it is not necessary to make separate volume measurements. With the simple relationship between the density of the sample and the natural frequency of the filled oscillator, it is possible to use the method for determining the density of samples which are injected into or flowing through the oscillator.

For the density calculation, we may consider a system represented by a hollow body of mass $M$ which is suspended on a spring with elasticity constant $c$, and whose volume $V$ is filled with sample of density $\rho$. The measured oscillation period $T$ of the entire system can be then used to calculate the sample density as (Kratky et al. 1973)

$\rho=\frac{c T^{2}-4 \pi^{2} M}{4 \pi^{2} V}$

Examples of the density measurements as function of temperature are shown in Fig. 2.

\section{Small angle neutron scattering}

SANS measurements were performed at YuMO, the time-offlight instrument (Kuklin et al. 2011b) at the IBR-2 pulsed nuclear reactor (JINR, Dubna, Russia) (Kuklin et al. 2011a). The beam of neutrons with wavelengths $\lambda$ varying from 0.5 to $8 \AA$ was formed by a set of two pinhole collimators of diameters 40 and $14 \mathrm{~mm}$ and brought onto the sample. The neutrons scattered by the sample were detected with two circular wire detectors placed at distances 4.5 and $13 \mathrm{~m}$. This experimental setup allows to cover a $q$-range of $(0.006$ $\left.\AA^{-1}, 0.5 \AA^{-1}\right)$, where $q=(4 \pi / \lambda) \sin \theta$ with $2 \theta$ representing the scattering angle. A vanadium standard was used for the calibration of absolute scattered intensity, while silver behenate sample was used to calibrate distances (NyamOsor et al. 2012). Raw data treatment was performed by the SAS software (Soloviev et al. 2017). The liquid samples were contained in 1-mm-thick flat quartz cuvettes (Hellma) and held in the multipositional sample holder connected to the

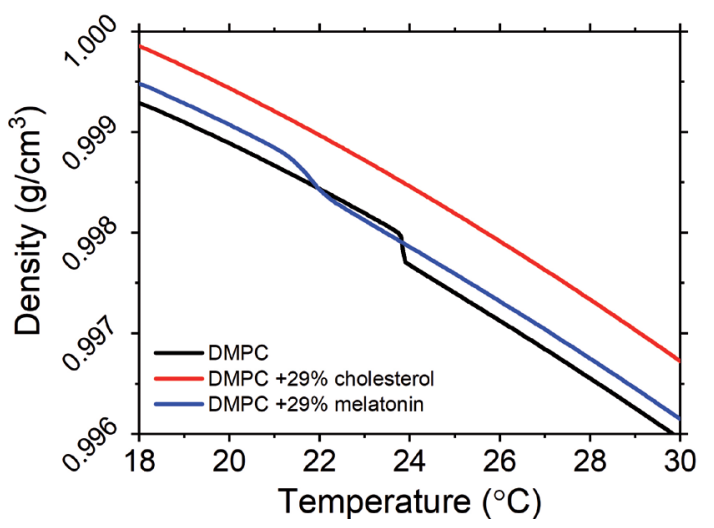

Figure 2. Densitometry curves measured for DMPC, DMPC+cholesterol (29 mol\%), and DMPC+melatonin (29 mol\%) multilamellar vesicles dispersed in $\mathrm{H}_{2} \mathrm{O}$. 
liquid thermostat Lauda with temperature controller Pt-100 at $25^{\circ} \mathrm{C}$. The obtained scattering curves were corrected for background scattering from the buffer solution.

The final SANS curves were fitted with small angle KratkyPorod approximation for disoriented lamellar objects (Feigin and Svergun 1987; Pencer and Hallett 2000):

$I(q)=\frac{A}{q^{2}} \cdot e^{-q^{2} \cdot R_{g}^{2}}+$ background

In this, $A$ is a constant depending on the average scattering density of lamella, its volume and quantity of lamellae in the sample, and $R_{g}$ is a radius of gyration along the thickness of lamella. The latter can be transformed for homogeneous distribution of scattering density into the thickness of lamella as $d_{g}=\sqrt{ } 12 \cdot R_{g}$. We choose this function for its simplicity and unambiguity. The shape of the theoretical scattering curve presented by this function depends only on one parameter $R_{g}$, while does not depend on the size of overall vesicle and inhomogeneity inside a membrane - these characteristics are included in parameters $A$ and $R_{g}$. The examples of SANS curves measured experimentally, and results of fitting procedure described above are presented in Fig. 3.

\section{Results and Discussion}

\section{Modulation of phase transition temperature}

The membrane thermodynamics depends mostly on its composition and temperature. Especially the latter is a wellknown and described determinant of the thermodynamic phase of lipid systems (Tristram-Nagle and Nagle 2004). Single lipids, or mixtures of lipids are characteristic to have a melting temperature at which they change from gel to

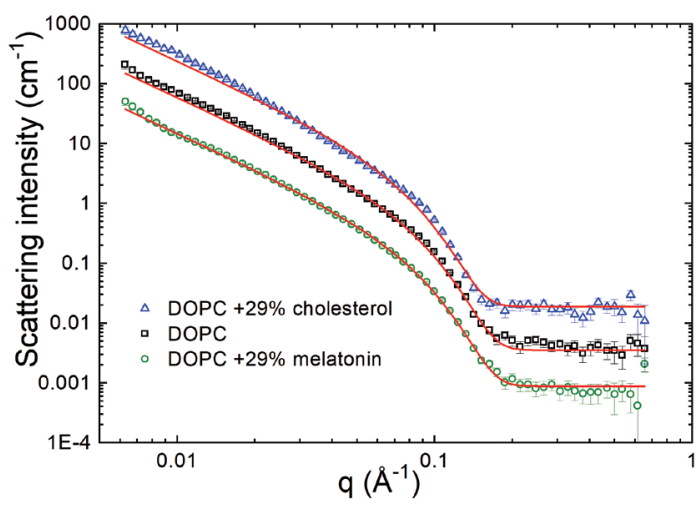

Figure 3. SANS curves for DOPC unilamellar vesicles doped with cholesterol or melatonin and dispersed in $100 \% \mathrm{D}_{2} \mathrm{O}$. Solid lines represent the Kratky-Porod approximation fits to the experimental data (open symbols). The curves are shifted vertically for better visualization (the multiplication factor of 4).

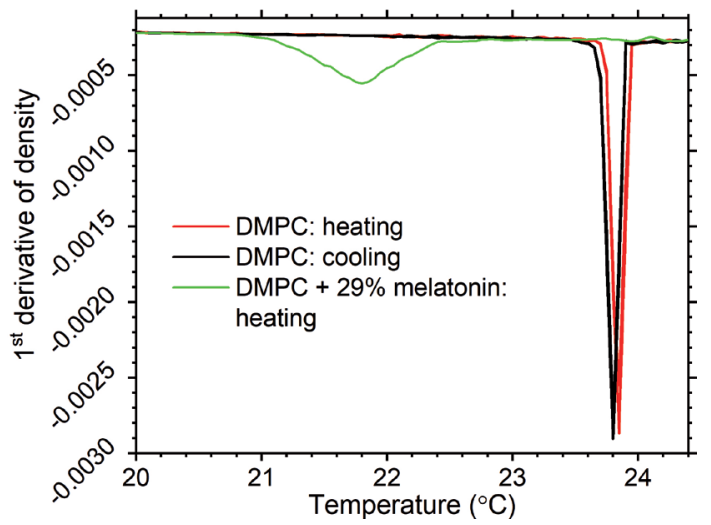

Figure 4. First derivatives of density changes as a function of temperature for neat DMPC multilayers (black and red curves corresponding to cooling and heating, respectively), and DMPC multilayers doped with $29 \mathrm{~mol} \%$ melatonin (green line).

fluid phase (the border may be more or less sharp depending on the complexity of system). The value of this phase change temperature is specific to each lipid species. On the other hand, it can be modulated, among other agents, by the additives. The fluidizing agents decrease the melting temperature, thus increase the propensity of lipid molecules to form fluid membranes. We have examined the effect of the two additives in our study employing the densitometry measurements.

First, the single lipid system consisting of MLVs dispersed in $\mathrm{H}_{2} \mathrm{O}$ was constructed and measured. DMPC was chosen for these measurements due to its melting temperature being conveniently accessible at $\mathrm{T}_{\mathrm{m}}=24.0^{\circ} \mathrm{C}$ (Nagle and Wilkinson 1978). Our measurements show very good reproducibility over the time of 6 measurement cycles ( $\mathrm{T}$ changing between 18 and $30^{\circ} \mathrm{C}$ ), and directions of the temperature changes (cooling vs. heating). We determine the phase transition temperature from the $1^{\text {st }}$ derivative of density changes to be 23.80-23.85 ${ }^{\circ} \mathrm{C}$ (Fig. 4 ).

Next, we have examined systems doped with cholesterol or melatonin. The addition of melatonin clearly changed the sharpness and position of the melting temperature to be broader and at a lower value. The first derivatives of density changes reveal $\mathrm{T}_{\mathrm{m}}=21.8^{\circ} \mathrm{C}$ (Fig. 4 ). It indicates the fluidizing effect of melatonin by shifting the membrane thermodynamics towards fluid phase, well in agreement with literature results (Severcan et al. 2005). This corroborates nicely the previous results concluding the disordering effect of melatonin on the lipid hydrocarbon chains (Drolle et al. 2013). The impact is most likely due to the incorporation of melatonin in the head group region introducing the defects and/or free volumes in the hydrocarbon region, that in turn increase the conformational space for hydrocarbon chains filling up the defects. 
In the case when doping the DMPC membrane with cholesterol, we have not been able to detect a sharp transition. This is very likely due to a well-known effect of cholesterol on the saturated hydrocarbon chains (Vist and Davis 1990). The gel phase lipid chains transition with increasing cholesterol concentration into new thermodynamic phase termed liquid ordered $\left(\mathrm{L}_{\mathrm{O}}\right)$, which transitions into liquid disordered phase $\left(\mathrm{L}_{\mathrm{d}}\right)$ with increasing temperature. The system behavior of binary and/or ternary mixtures becomes complex with phase diagrams including regions where various phases coexist, and phase boundaries get smeared (Davis and Schmidt 2019). Although the densitometric measurements may not be capable detecting the phase changes in systems at high cholesterol concentrations, the cholesterol's ordering effect on the lipid chains has been documented in many studies. It is apparently best evident through the decreased lateral area per lipid and consequently the increased bilayer thickness (Rog et al. 2009). We thus turn to scrutinize the bilayer thickness in further.

\section{Modulation of membrane thickness}

Membrane fluidity is an indicative of the dynamics of membrane components, consisting of rotational and lateral diffusion, and most importantly, the hydrocarbon chain conformational changes (Wanderlingh et al. 2017). The less fluid membrane is characteristic of slower diffusion and a high ratio of all-trans chains that have a minimal freedom in conformational changes. Consequently, the chain length appears at its maximum and so does the thickness of lipid bilayer. On the other hand, fluid membranes comprise hydrocarbon chains with frequent trans-gauche conformational changes due to which the projected chain length decreases. It is therefore possible to appraise the membrane fluidity and its changes by measuring the thickness of membrane in the direction along its normal. We evaluate the effect of cholesterol and/or melatonin based on membrane thickness changes as measured by SANS. A single lipid membrane model was selected for simplicity while eradicating effects of complex systems. DOPC lipid was chosen for the presence of biologically relevant double bonds.

We assess the dependence of the thickness parameter $d_{g}$ of DOPC membrane upon the addition of cholesterol first. In the approximation of homogeneous membrane, its thickness increases from $44.0 \pm 0.7 \AA$ (pure DOPC) to $47.5 \pm 1.0 \AA$ (DOPC $+29 \%$ cholesterol). It is worth to note, that our measurements performed with hydrogenrich lipid bilayers dispersed in $100 \% \mathrm{D}_{2} \mathrm{O}$ ensure the best conditions for the mentioned approximation. Nevertheless, the extracted values should be understood in the context of the approximation and not in terms of absolute values that proved to be slightly different when utilizing much more sophisticated evaluation approaches (Kučerka et al. 2008a). In the present work on the other hand, we study the tendency of changing the membrane thickness without a need for precise absolute values, and focus therefore on the thickness differences (Fig. 5).

The effect of membrane thickening upon the cholesterol incorporation is well known and connected with so-called 'condensing effect' that includes increasing of the ordering of the lipid tails and consequently their elongation. The cholesterol molecules are found to be located in the hydrocarbon region of the bilayer aligned parallel to the lipid tails, the hydrophilic cholesterol part is located at the phospholipid heads. This effect has been described in experiments with model membranes (Rappolt et al. 2003; Gallová et al. 2004; Pencer et al. 2005; Kučerka et al. 2008b; Drolle et al. 2013; Hung et al. 2016) and by computer simulations (Smondyrev and Berkowitz 1999; Chiu et al. 2002; Jedlovszky and Mezei 2003; Leeb and Maibaum 2018; Rivel et al. 2019), and is in a perfect agreement with our densitometric and SANS results.

Intriguingly, our present results extend the condensing effect of cholesterol on the melatonin-including membranes. We have observed the bilayer thickening due to cholesterol in the cases where various amounts of melatonin (up to $29 \mathrm{~mol} \%$ ) have been mixed with DOPC. In all of the systems studied, approximately the same relative changes to the membrane thickness have been observed, although a minor decrease of the tendency can be noticed (Fig. 5). The interactions between cholesterol and melatonin do not appear to modulate the impact of cholesterol itself on lipid bilayers.

Further, we evaluate the effect of melatonin. As can be seen from Fig. 6, melatonin added at various concentrations (up to $29 \mathrm{~mol} \%$ ) causes a decrease in the bilayer thickness parameter $d_{g}$ by $0.6 \AA$. Its impact on the cholesterol-loaded

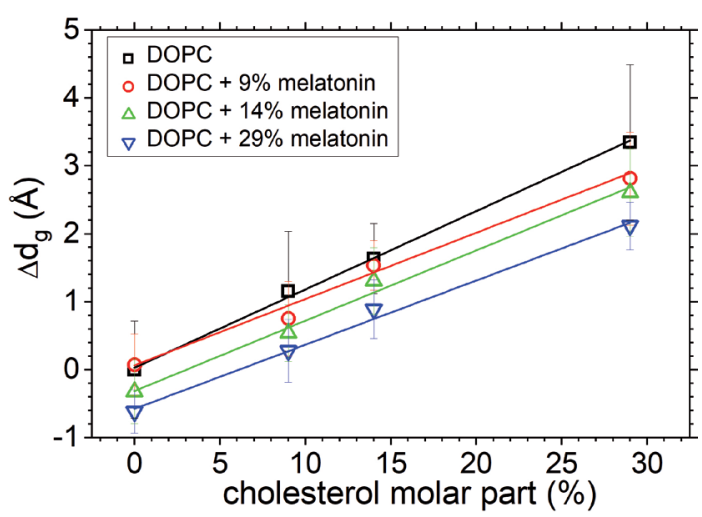

Figure 5. The impact of cholesterol on the bilayer thickness parameter $d_{g}$ of unilamellar vesicles made of DOPC, and DOPC with various amounts of melatonin added. $\Delta d_{g}$ is shown relative to the $d_{g}$ of neat DOPC bilayer. The solid lines are linear fits to guide the eyes. 


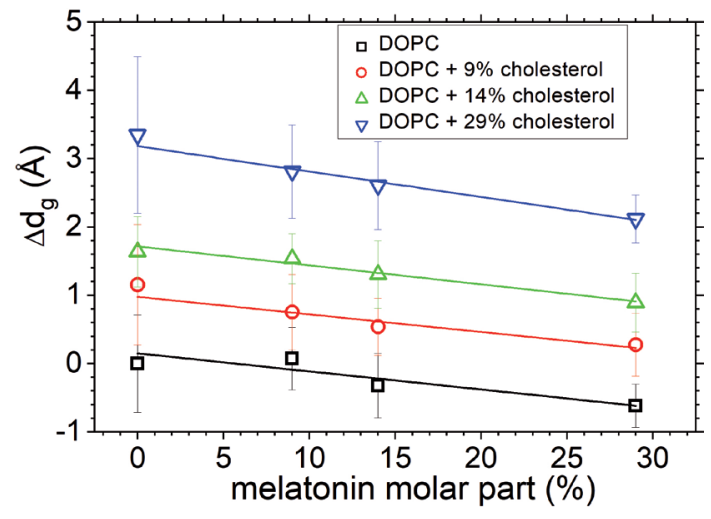

Figure 6. The impact of melatonin on the bilayer thickness parameter $d_{g}$ of unilamellar vesicles made of DOPC, and DOPC with various amounts of cholesterol added. $\Delta d_{g}$ is shown relative to the $d_{g}$ of neat DOPC bilayer. The solid lines are linear fits to guide the eyes.

DOPC bilayers appears to be very similar (Fig. 6), in the agreement with the above discussed effect of cholesterol on the melatonin-loaded bilayers. The mutual interactions between cholesterol and melatonin do not appear to play a significant role, unlike the interactions between lipid bilayers and cholesterol or melatonin themselves. It is worthwhile to note smaller impact of melatonin on the lipid bilayer compared to that of cholesterol. This can, however, be explained by relatively smaller molecule of melatonin (its molecular mass is only $232.28 \mathrm{~g} / \mathrm{mol}$ compared to $386.65 \mathrm{~g} / \mathrm{mol}$ in the case of cholesterol (Sigma-Aldrich)), which therefore leads to the smaller disturbance of membrane.

Our observations are consistent again with experimental and simulated results presented previously (Drolle et al. 2013). The effect of melatonin can be connected with the disordering of lipid tails promoted by melatonin. The molecules of melatonin are located near phospholipid head groups and lead to a distance expansion between lipid heads. Consequently, the hydrocarbon region expands laterally, while decreases in the direction along the bilayer normal (i.e., bilayer thickness). This enlarges the conformational space of lipid tails resulting into the decrease of their order parameter. The ensuing effect of melatonin concludes nicely its fluidizing effect as observed in our densitometric and SANS measurements.

The thinning of membrane in a presence of low melatonin concentrations has been detected also by two-dimensional $\mathrm{x}$-ray diffraction (Dies et al. 2015) and for monolayers by Brewster angle microscopy (De Lima et al. 2010). Changes in physico-chemical properties and ordering of bilayer in presence of melatonin have been demonstrated in various works too (Saija et al. 2002; Sahin et al. 2007; De Lima et al. 2010; Drolle et al. 2013; Choi et al. 2014; Dies et al. 2015). We can conclude a full agreement of our experimental results with those published previously, and importantly, extend them to the cases of mutual incorporation with cholesterol. We find unambiguously the two molecules impacting the lipid bilayers additively in opposing directions, i.e., cholesterol in a role of condensing agent, while melatonin as a fluidizing agent. In the context of potential importance of membrane fluidity in the offset of various conformational diseases, it is important to explore further the interactions of amyloidbeta peptides with such platforms constructed at controlled levels of fluidity.

\section{Presence of amyloid-beta peptides}

We examine the effect of membrane fluidity as controlled via the addition of cholesterol or melatonin further in the case $\mathrm{A} \beta_{25-35}$ peptide is present in the membrane. The choice for selecting these segments was motivated by the fact this is the shortest $\mathrm{A} \beta$ fragment retaining a high cytotoxicity (Millucci et al. 2010). It is worth noting that literature results are yet to come to the satisfactory conclusions regarding the location of various $A \beta$ fragments, including $A \beta_{25-35}$, relative to lipid membrane (Ermilova and Lyubartsev 2020). Our present results do not allow us to make direct conclusions on the issue either. Another intriguing information, however, is the impact of the peptide itself on the structure of the membrane (Jelinek 2018). We therefore survey our various systems discussed above for the change of membrane thickness upon the addition of $\mathrm{A} \beta_{25-35}$ peptide.

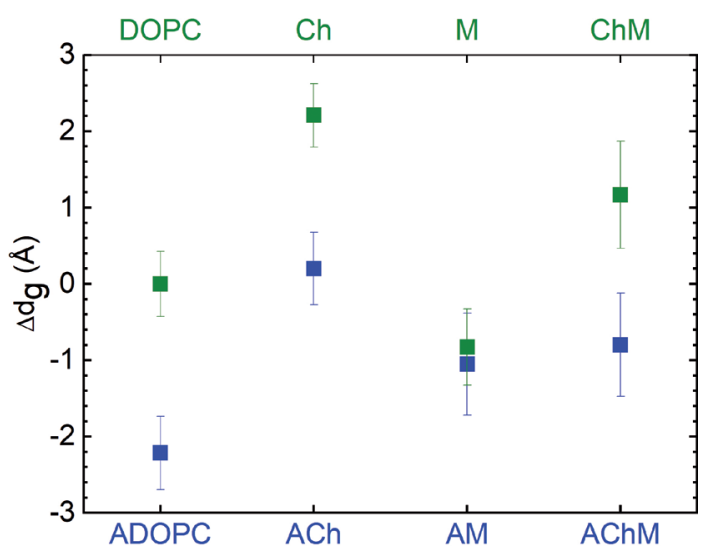

Figure 7. The changes to bilayer thickness parameter $d_{g}$ of various membranes studied upon the addition of $A \beta_{25-35}$ peptide. The green points (top axis) show the results for neat DOPC, and DOPC with $29 \mathrm{~mol} \%$ cholesterol (Ch), 29 mol\% melatonin (M) and both $29 \mathrm{~mol} \%$ cholesterol and $29 \mathrm{~mol} \%$ melatonin (ChM). The blue points (bottom axis) show the results for the corresponding systems with additional $3 \mathrm{~mol} \%$ of $\mathrm{A} \beta_{25-35}$ peptide (i.e., ADOPC, ACh, AM, AChM). $\Delta d_{g}$ is shown relative to the $d_{g}$ of neat DOPC bilayer. 
The addition of peptide at the level of $3 \mathrm{~mol} \%$ assumes a minimal disturbance to the underlying system (Dies et al. 2014). Nevertheless, we have observed in some of our experimental measurements a small quasi-Bragg peak at q 0.1 $\AA^{-1}$ in addition to the smooth SANS curve (data not shown). Such peak appears due to a long-range order, suggesting that samples with $\mathrm{A} \beta$ peptide added are more prone to inter-bilayer interactions. The Kratky-Porod approximation analysis was though applied to the peak free SANS curves only that we obtained in the case of ULVs dispersed in $75 \% \mathrm{D}_{2} \mathrm{O}$. The results shown in Fig. 7 then suggest a significant thinning of the DOPC bilayer upon the addition of peptide (compare the results denoted by DOPC and ADOPC corresponding to the neat DOPC bilayers and those with the addition of $A \beta$ peptide, respectively). Our observation corroborates clearly the interaction of peptide with the underlying lipid bilayer. Apparently, it increases the disorder of hydrocarbon chains resulting to the shortening of their projected length.

The disorder increase born by additives in lipid membranes is typically triggered by their implementation in lipid head group region, thus by increasing the lateral area per lipid. Such location of $A \beta_{25-35}$ peptide has been indeed reported (Dies et al. 2014). Further, elevated concentrations of cholesterol in lipid bilayers constructed from saturated or mixed saturated-monounsaturated lipids with partially negatively-charged head groups, and measured at various dehydrated phases also revealed the peptide adsorption rather than its insertion (Dante et al. 2006). This is in consent with our results that show a peptide-triggered thinning of membrane containing 29 mol\% cholesterol (Fig. 7, results denoted by $\mathrm{Ch}$ for bilayers with cholesterol and $\mathrm{ACh}$ for bilayers with $\mathrm{A} \beta$ peptide and cholesterol). The effect of peptide appears to suggest again the increase of lipid chains disorder caused, most likely, by its incorporation at lipid-water interface.

The situation is different in the case of melatonin-loaded membranes. Very intriguingly, the thickness change becomes negligibly small when comparing membranes without or with peptide addition (Fig. 7, results denoted by M and AM, respectively). Unlike in the other two systems discussed above, melatonin containing membranes do not respond to the addition of peptide by the increased disorder. We conclude this to be possibly a result of peptide incorporation away from lipid head group region and thus within their hydrocarbon chains. Literature reports such orientation of peptide while interacting with membranes that contained melatonin, though to a much smaller extent (Dies et al. 2014). The differences between underlying membrane systems reported previously and in our case (e.g., chain saturation and length, head group charge), perhaps account for concluded differences in the peptide distribution.

Finally, we examine the system with a concurrent addition of cholesterol and melatonin. While the comparison of peptide-free membranes discussed in the previous section reveals clearly the effect being intermediate between those caused by cholesterol or melatonin separately, the additiveness is much more subtle in the case of peptide-added membrane. Figure 7 (results denoted by ChM and AChM for the DOPC bilayers containing cholesterol and melatonin without or with $\mathrm{A} \beta$ peptide addition, respectively) suggests similarly significant decrease of membrane thickness upon the peptide addition as in the cases of neat DOPC bilayers and those loaded with cholesterol. A small reduction can nevertheless be noted, that may suggest a portion of peptide being incorporated within the lipid bilayer core. This would be in line with the above concluded impact of the fluidizing effect of melatonin. Nonetheless, our present results do not allow us to make a direct observation of the peptide location. The unambiguous changes to the membrane thickness detected in this study on the other hand serve an intriguing base for further studies focusing on the interactions between amyloid-beta peptides and membranes with a tweakable fluidity.

\section{Conclusions}

We have researched the impact of cholesterol and melatonin on the structure and dynamics of simple model membrane constructed from DOPC. While cholesterol results clearly into membrane thickening, melatonin causes its thinning. This is linked to the fluidity of membrane, and scrutinized further to reveal its role in the bilayer-peptide interactions. Considering the membrane thickness decrease upon the addition of $\mathrm{A} \beta_{25-35}$ peptide, we conclude its location in the lipid head group region for neat DOPC bilayers and those comprising cholesterol. The results for the system of DOPC bilayers with melatonin on the other hand, suggest the incorporation of peptide within the lipid bilayer core. We propose the redistribution of peptide arrangement to be promoted by the fluidizing effect of melatonin.

Acknowledgement. This work has been supported by the Russian Science Foundation under grant 19-72-20186.

\section{References}

Ahyayauch H, Raab M, Busto JV, Andraka N, Arrondo JLR, Masserini M, Tvaroska I, Goni FM (2012): Binding of beta-amyloid (1-42) peptide to negatively charged phospholipid membranes in the liquid-ordered state: modeling and experimental studies. Biophys. J. 103, 453-463

https://doi.org/10.1016/j.bpj.2012.06.043

Barrett MA, Alsop RJ, Hauss T, Rheinstadter MC (2015): The position of abeta22-40 and abeta 1-42 in anionic lipid membranes containing cholesterol. Membranes 5, 824-843 https://doi.org/10.3390/membranes5040824 
Ceraulo L, Ferrugia M, Tesoriere L, Segreto S, Livrea MA, Turco Liveri V (1999): Interactions of melatonin with membrane models: portioning of melatonin in AOT and lecithin reversed micelles. J. Pineal Res. 26, 108-112 https://doi.org/10.1111/j.1600-079X.1999.tb00570.x

Chiu SW, Jakobsson E, Mashl RJ, Scott HL (2002): Cholesterolinduced modifications in lipid bilayers: A simulation study. Biophys. J. 83, 1842-1853 https://doi.org/10.1016/S0006-3495(02)73949-0

Choi Y, Attwood SJ, Hoopes MI, Drolle E, Karttunen M, Leonenko Z (2014): Melatonin directly interacts with cholesterol and alleviates cholesterol effects in dipalmitoylphosphatidylcholine monolayers. Soft Matter. 10, 206-213 https://doi.org/10.1039/C3SM52064A

Chu N, Kučerka N, Liu Y, Tristram-Nagle S, Nagle JF (2005): Anomalous swelling of lipid bilayer stacks is caused by softening of the bending modulus. Phys. Rev. E 71, 041904 https://doi.org/10.1103/PhysRevE.71.041904

Dante S, Hauss T, Dencher NA (2006): Cholesterol inhibits the insertion of the Alzheimer's peptide A beta(25-35) in lipid bilayers. Eur. Biophys. J. 35, 523-531 https://doi.org/10.1007/s00249-006-0062-x

Davis JH, Schmidt ML (2019): Cholesterol in model membranes. In: Characterization of Biological Membranes: Structure and Dynamics (Eds. MP Nieh, FA Heberle, J Katsaras), pp. 325364, Berlin https://doi.org/10.1515/9783110544657-010

De Lima VR, Caro MSB, Munford ML, Desbat B, Dufourc E, Pasa AA, Creczynski-Pasa TB (2010): Influence of melatonin on the order of phosphatidylcholine-based membranes. J. Pineal Res. 49, 169-175 https://doi.org/10.1111/j.1600-079X.2010.00782.x

Dies H, Toppozini L, Rheinstadter MC (2014): The interaction between amyloid-beta peptides and anionic lipid membranes containing cholesterol and melatonin. PLoS One 9, e99124 https://doi.org/10.1371/journal.pone.0099124

Dies H, Cheung B, Tang J, Rheinstädter MC (2015): The organization of melatonin in lipid membranes. Biochim. Biophys. Acta 1848, $1032-1040$ https://doi.org/10.1016/j.bbamem.2015.01.006

Drolle E, Kučerka N, Hoopes MI, Choi Y, Katsaras J, Karttunen M, Leonenko Z (2013): Effect of melatonin and cholesterol on the structure of DOPC and DPPC membranes. Biochim. Biophys. Acta 1828, 2247-2254 https://doi.org/10.1016/j.bbamem.2013.05.015

Ermilova I, Lyubartsev AP (2019): Cholesterol in phospholipid bilayers: positions and orientations inside membranes with different unsaturation degrees. Soft Matter. 15, 78-93 https://doi.org/10.1039/C8SM01937A

Ermilova I, Lyubartsev AP (2020): Modelling of interactions between $A \beta(25-35)$ peptide and phospholipid bilayers: effects of cholesterol and lipid saturation. RSC Advances 7 https://doi.org/10.1039/C9RA06424A

Feigin LA, Svergun DI (1987): Structure Analysis by Small-Angle $\mathrm{X}$-Ray and Neutron Scattering. Springer USA https://doi.org/10.1007/978-1-4757-6624-0

Gallová J, Uhríková D, Hanulová M, Teixeira J, Balgavý P (2004): Bilayer thickness in unilamellar extruded 1,2-dimyristoleoyl and 1,2-dierucoyl phosphatidylcholine vesicles: SANS contrast variation study of cholesterol effect. Colloids Surf. B Biointerfaces 38, 11-14 https://doi.org/10.1016/j.colsurfb.2004.07.012

Huang J, Buboltz JT, Feigenson GW (1999): Maximum solubility of cholesterol in phosphatidylcholine and phosphatidylethanolamine bilayers. Biochim. Biophys. Acta 1417, 89-100 https://doi.org/10.1016/S0005-2736(98)00260-0

Hung W-C, Lee M-T, Chung H, Sun Y-T, Chen H, Charron Nicholas E, Huang Huey W (2016): Comparative study of the condensing effects of ergosterol and cholesterol. Biophys. J. 110, 2026-2033 https://doi.org/10.1016/j.bpj.2016.04.003

Jedlovszky P, Mezei M (2003): Effect of cholesterol on the properties of phospholipid membranes. 1. Structural features. J. Phys. Chem. B. 107, 5311-5321 https://doi.org/10.1021/jp0219505

Jelinek R (2018): Membranes, From Biological Functions to Therapeutic Applications. De Gruyter https://doi.org/10.1515/9783110453690

Karasek M (2004): Melatonin, human aging, and age-related diseases. Exp. Gerontol. 39, 1723-1729 https://doi.org/10.1016/j.exger.2004.04.012

Kratky O, Leopold H, Stabinger H (1973): The determination of the partial specific volume of proteins by the mechanical oscillator technique. Methods Enzymol. 27, 98-110 https://doi.org/10.1016/S0076-6879(73)27007-6

Kučerka N, Pencer J, Sachs JN, Nagle JF, Katsaras J (2007): Curvature effect on the structure of phospholipid bilayers. Langmuir 23, 1292-1299 https://doi.org/10.1021/la062455t

Kučerka N, Nagle JF, Sachs JN, Feller SE, Pencer J, Jackson A, Katsaras J (2008a): Lipid bilayer structure determined by the simultaneous analysis of neutron and X-ray scattering data. Biophys. J. 95, 2356-2367 https://doi.org/10.1529/biophysj.108.132662

Kučerka N, Perlmutter JD, Pan J, Tristram-Nagle S, Katsaras J, Sachs JN (2008b): The effect of cholesterol on short- and long-chain monounsaturated lipid bilayers as determined by molecular dynamics simulations and X-ray scattering. Biophys. J. 95, 2792-2805 https://doi.org/10.1529/biophysj.107.122465

Kučerka N, Marquardt D, Harroun TA, Nieh MP, Wassall SR, Katsaras J (2009): The functional significance of lipid diversity: orientation of cholesterol in bilayers is determined by lipid species. J. Am. Chem. Soc. 131, 16358-16359 https://doi.org/10.1021/ja907659u

Kučerka N, Heberle FA, Pan J, Katsaras J (2015): Structural significance of lipid diversity as studied by small angle neutron and X-ray scattering. Membranes 5, 454-472 https://doi.org/10.3390/membranes5030454

Kuklin AI, Rogov AD, Gorshkova YE, Utrobin PK, Kovalev YS, Rogachev AV, Ivankov OI, Kutuzov SA, Soloviov DV, Gordeliy VI (2011a): Analysis of neutron spectra and fluxes obtained with cold and thermal moderators at IBR-2 reactor: Experimental and computer-modeling studies. Phys. Part At. Nucl. Lett. 8, 119 https://doi.org/10.1134/S1547477111020075 
Kuklin AI, Soloviev DV, Rogachev AV, Utrobin PK, Kovalev YS, Balasoiu M, Ivankov OI, Sirotin AP, Murugova TN, Petukhova TB, et al. (2011b): New opportunities provided by modernized small-angle neutron scattering two-detector system instrument (YuMO). J. Phys. Conf. 291, 012013 https://doi.org/10.1088/1742-6596/291/1/012013

Leeb F, Maibaum L (2018): Spatially resolving the condensing effect of cholesterol in lipid bilayers. Biophys. J. 115, 2179-2188 https://doi.org/10.1016/j.bpj.2018.10.024

Lim L, Wenk MR (2009): Neuronal membrane lipids - their role in the synaptic vesicle cycle. In: Handbook of Neurochemistry and Molecular Neurobiology: Neural Lipids (Eds. A Lajtha, G Tettamanti, G Goracci), pp. 223-238, Boston, MA https://doi.org/10.1007/978-0-387-30378-9_9

Martel A, Antony L, Gerelli Y, Porcar L, Fluitt A, Hoffmann K, Kiesel I, Vivaudou M, Fragneto G, de Pablo JJ (2017): Membrane permeation versus amyloidogenicity: a multitechnique study of islet amyloid polypeptide interaction with model membranes. J. Am. Chem. Soc. 139, 137-148 https://doi.org/10.1021/jacs.6b06985

Martinez-Seara H, Rog T, Pasenkiewicz-Gierula M, Vattulainen I, Karttunen M, Reigada R (2008): Interplay of unsaturated phospholipids and cholesterol in membranes: effect of the double-bond position. Biophys. J. 95, 3295-3305 https://doi.org/10.1529/biophysj.108.138123

Millucci L, Ghezzi L, Bernardini G, Santucci A (2010): Conformations and biological activities of amyloid beta peptide 25-35. Curr. Protein Pept. Sci. 11, 54-67 https://doi.org/10.2174/138920310790274626

Murphy RM (2007): Kinetics of amyloid formation and membrane interaction with amyloidogenic proteins. Biochim. Biophys. Acta 1768, 1923-1934 https://doi.org/10.1016/j.bbamem.2006.12.014

Nagle JF, Tristram-Nagle S (2000): Structure of lipid bilayers. Biochim. Biophys. Acta 1469, 159-195 https://doi.org/10.1016/S0304-4157(00)00016-2

Nagle JF, Wilkinson DA (1978): Lecithin bilayers. Density measurement and molecular interactions. Biophys. J. 23, 159-175 https://doi.org/10.1016/S0006-3495(78)85441-1

Ntarakas N, Ermilova I, Lyubartsev AP (2019): Effects of lipid saturation on amyloid-beta peptides partitioning and aggregation in neuronal membranes: molecular dynamics simulations. Eur. Biophys. J. 48, 813-824 https://doi.org/10.1007/s00249-019-01407-x

Nyam-Osor M, Soloviov DV, Kovalev YS, Zhigunov A, Rogachev AV, Ivankov OI, Erhan RV, Kuklin AI (2012): Silver behenate and silver stearate powders for calibration of SAS instruments. J. Phys. Conf. 351, 012024 https://doi.org/10.1088/1742-6596/351/1/012024

Pencer J, Hallett FR (2000): Small-angle neutron scattering from large unilamellar vesicles: An improved method for membrane thickness determination. Phys. Rev. E. 61, 3003-3008 https://doi.org/10.1103/PhysRevE.61.3003

Pencer J, Nieh M-P, Harroun TA, Krueger S, Adams C, Katsaras J (2005): Bilayer thickness and thermal response of dimyristoylphosphatidylcholine unilamellar vesicles containing cholesterol, ergosterol and lanosterol: A small-angle neutron scattering study. Biochim. Biophys. Acta 1720, 84-91 https://doi.org/10.1016/j.bbamem.2005.10.017

Petrache HI, Gouliaev N, Tristram-Nagle S, Zhang RT, Suter RM, Nagle JF (1998): Interbilayer interactions from high-resolution x-ray scattering. Phys. Rev. E 57, 7014-7024 https://doi.org/10.1103/PhysRevE.57.7014

Prince M, Comas-Herrera A, Knapp M, Guerchet M, Karagiannidou M (2016): World Alzheimer report 2016: Improving healthcare for people living with dementia: Coverage, quality and costs now and in the future. Alzheimer's Disease International, London, UK.

Rappolt M, Vidal M, Kriechbaum M, Steinhart M, Amenitsch H, Bernstorff S, Laggner P (2003): Structural, dynamic and mechanical properties of POPC at low cholesterol concentration studied in pressure/temperature space. Eur. Biophys. J. 31, 575-585 https://doi.org/10.1007/s00249-002-0253-z

Reiter RJ, Tan DX, Galano A (2014): Melatonin reduces lipid peroxidation and membrane viscosity. Front. Physiol. 5, 377 https://doi.org/10.3389/fphys.2014.00377

Rivel T, Ramseyer C, Yesylevskyy S (2019): The asymmetry of plasma membranes and their cholesterol content influence the uptake of cisplatin. Sci. Rep. 9, 5627-5627 https://doi.org/10.1038/s41598-019-41903-w

Rog T, Pasenkiewicz-Gierula M, Vattulainen I, Karttunen M (2009): Ordering effects of cholesterol and its analogues. Biochim. Biophys. Acta 1788, 97-121 https://doi.org/10.1016/j.bbamem.2008.08.022

Sahin I, Severcan F, Kazanc1 N (2007): Melatonin induces opposite effects on order and dynamics of anionic DPPG model membranes. J. Mol. Struct. 834-836, 195-201 https://doi.org/10.1016/j.molstruc.2006.12.002

Saija A, Tomaino A, Trombetta D, Pellegrino ML, Tita B, Caruso S, Castelli F (2002): Interaction of melatonin with model membranes and possible implications in its photoprotective activity. Eur. J. Pharm. Biopharm. 53, 209-215 https://doi.org/10.1016/S0939-6411(01)00239-9

Severcan F, Sahin I, Kazanci N (2005): Melatonin strongly interacts with zwitterionic model membranes--evidence from Fourier transform infrared spectroscopy and differential scanning calorimetry. Biochim. Biophys. Acta 1668, 215-222 https://doi.org/10.1016/j.bbamem.2004.12.009

Smondyrev AM, Berkowitz ML (1999): Structure of dipalmitoylphosphatidylcholine/cholesterol bilayer at low and high cholesterol concentrations: Molecular dynamics simulation. Biophys. J. 77, 2075-2089 https://doi.org/10.1016/S0006-3495(99)77049-9

Soloviev AG, Solovjeva TM, Ivankov OI, Soloviov DV, Rogachev AV, Kuklin AI (2017): SAS program for two-detector system: seamless curve from both detectors. J. Phys. Conf. 848, 012020 https://doi.org/10.1088/1742-6596/848/1/012020

Tristram-Nagle S, Nagle JF (2004): Lipid bilayers: thermodynamics, structure, fluctuations, and interactions. Chem. Phys. Lipids 127, 3-14 https://doi.org/10.1016/j.chemphyslip.2003.09.002

Vist MR, Davis JH (1990): Phase equilibria of cholesterol/dipalmitoylphosphatidylcholine mixtures: $2 \mathrm{H}$ nuclear magnetic resonance and differential scanning calorimetry. Biochemistry 29, 451-464 https://doi.org/10.1021/bi00454a021 
Wanderlingh U, Branca C, Crupi C, Nibali VC, La Rosa G, Rifici S, Ollivier J, D’Angelo G (2017): Molecular dynamics of POPC phospholipid bilayers through the gel to fluid phase transition: An incoherent quasi-elastic neutron scattering study. Journal of Chemistry 2017, 3654237 https://doi.org/10.1155/2017/3654237

Yu H, Dickson EJ, Jung SR, Koh DS, Hille B (2016): High membrane permeability for melatonin. J. Gen. Physiol. 147, 63-76 https://doi.org/10.1085/jgp.201511526

Zhang J, Liu Q (2015): Cholesterol metabolism and homeostasis in the brain. Protein Cell. 6, 254-264

https://doi.org/10.1007/s13238-014-0131-3

Received: September 30, 2019

Final version accepted: November 17, 2019 